\title{
The European model for inhabited areas (ERMIN) - developing a description of the urban environment
}

\author{
T.W. CHARNOCK
}

\begin{abstract}
The European Model for Inhabited Areas (ERMIN) was developed to explore recovery options following the contamination of an urban environment with radioactive material. This paper discusses the user experience of ERMIN and in particular it concentrates on the development of a description of the urban environment and how that description affects the output. The user interacts with ERMIN by defining different regions on a map. A region may be an area in which a particular combination of recovery countermeasures is applied, or where a particular level of contamination is found, or where there is a particular type of urban environment. Most of the output from ERMIN is also presented as maps with summary information that allows the user to compare different countermeasure strategies. ERMIN has been implemented within two international nuclear accident Decision Support Systems; RODOS and ARGOS. Both systems have user interfaces that exploit geographic information system (GIS) technology which complement the ERMIN map based approach.
\end{abstract}

\section{Introduction}

The European Model for Inhabited Areas (ERMIN) was developed to allow users to explore different recovery options and to help develop and refine an appropriate strategy following the contamination of an urban environment with radioactive material. The input data include a description of the environment, initial deposition of radionuclides on to a reference surface and a description of countermeasure strategy that the user is considering. Output information includes the average effective doses to members of the public from external exposure to gamma and beta radiation from deposited radionuclides and inhalation of resuspended radionuclides, the contamination on urban surfaces, the activity concentration in air from resuspension, the doses to workers undertaking the recovery work, the quantity and activity of waste generated and the cost and work required to implement the countermeasure.

\footnotetext{
1 Health Protection Agency, Centre for Radiation, Chemical and Environmental Hazards, OX11 0RQ, Chilton, Didcot, OXON, UK.
} 
The technical and scientific details of ERMIN are given elsewhere (Charnock et al., 2009). This paper concentrates on the user experience, in particular on the development of a description of the urban environment and how that description affects the output.

\section{The user experience}

ERMIN has been implemented within two international nuclear accident Decision Support Systems: RODOS and ARGOS. Both systems have user interfaces that exploit geographic information system (GIS) technology and these complement the ERMIN approach that requires the user to set up an ERMIN run by defining regions on a map. A region may be an area in which a particular combination of recovery countermeasures is applied, where a particular level of contamination is found, or where there is a particular type of urban environment. Most of the output from ERMIN is also presented as maps with summary information that allows the user to compare different countermeasure strategies.

The first task a user of ERMIN is presented with is defining the scale and the resolution for ERMIN. The user defines the area of interest by drawing a rectangle on a map and specifying a grid square resolution. A reasonable scale at which to work would be from a few streets in a town with a grid square resolution of 100 by 100 metres to a scale of a few hundred kilometres encompassing a number of towns and cities with a resolution of $2 \mathrm{~km}$. Figure 1 shows the RODOS interface with an ERMIN grid covering a few tens of kilometres and resolution of $500 \mathrm{~m}$. Once a grid is set up, the user can then define regions by grouping grid squares together.

\section{Urban environment description}

Users often find that specifying the urban environment is the most challenging part of setting up an ERMIN run. In ERMIN the built environment is described by first grouping grid squares into regions with distinct environments and then assigning to each a mix of idealised environments from the database. For example a region maybe described as $100 \%$ "Semi-detached houses" or in a more complicated example 25\% "Open Area", 25\% "Terraced houses" and 50\% "multi-storey building".

Version one of the ERMIN environment database contains eight idealised environments (Tab. I) which were obtained from previous studies and not specifically developed for ERMIN. Consequently there are some weaknesses, and two in particular may confuse the user. Firstly two environments both confusingly represent semi-detached houses and the user is advised to ignore one and 


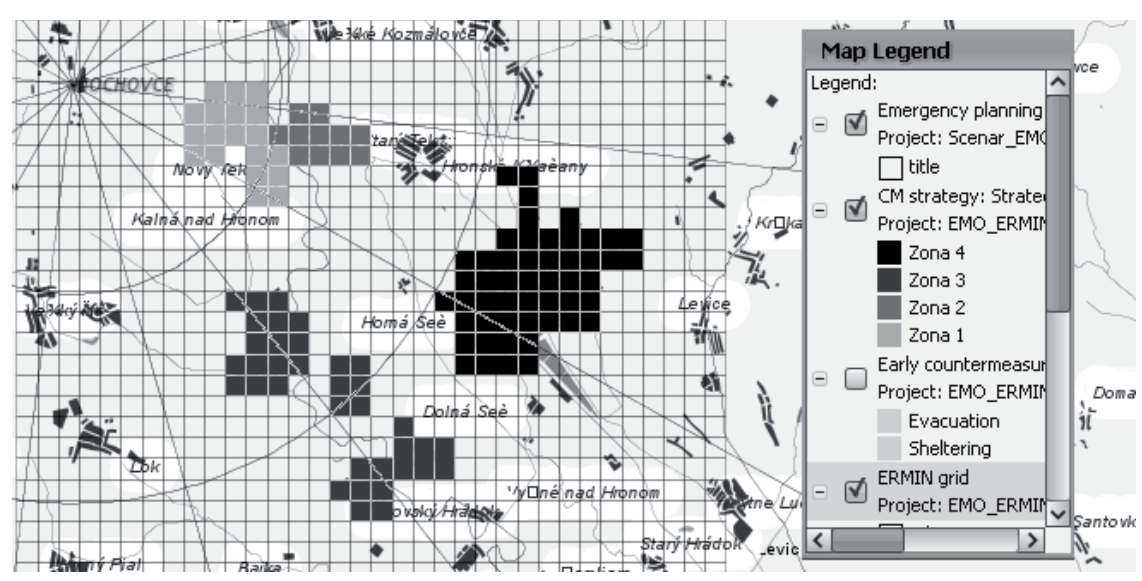

Figure 1 - The ERMIN interface in RODOS, the ERMIN grid has been specified to encompass a number of towns for a hypothetical incident in the Slovak republic. Environment descriptions have been entered for each of several nearby towns.

TABLE I

Environments in version 1 of the ERMIN database.

\begin{tabular}{ll}
\hline Street of detached prefabricated houses & Multi-storey block of flats amongst other house blocks \\
Street of semi-detached houses with basement & Multi-storey block of flats opposite parkland \\
Street of semi-detached houses without basement & Industrial site (Incomplete dose-rate library) \\
Street of terraced houses & Large open area \\
\hline
\end{tabular}

consistently use the other. Secondly, the "Industrial site" environment has an incomplete dose-rate library. It was included for interest and because in certain situations it may be useful, for example for planning purposes where the radionuclide involved may not be crucial. Despite these weaknesses the database can be used to describe a broad range of urban situations and it is hoped to be able to expand and consolidate it in future releases of ERMIN.

In order to specify the urban environment, the user needs to know the real environment; in particular the sizes of buildings, the amount of open space, the proportion of paved surfaces and the number of trees. Ideally the user will be familiar with the local environment or will be able to talk to someone who is. Maps can give visual clues to the type of environment, and internet map sites such as Google $^{\mathrm{TM}}$ or Bing ${ }^{\mathrm{TM}}$ provide access to aerial imagery at a sufficiently high resolution over much of Europe and other parts of the world, that individual houses, roads and trees can be identified. 


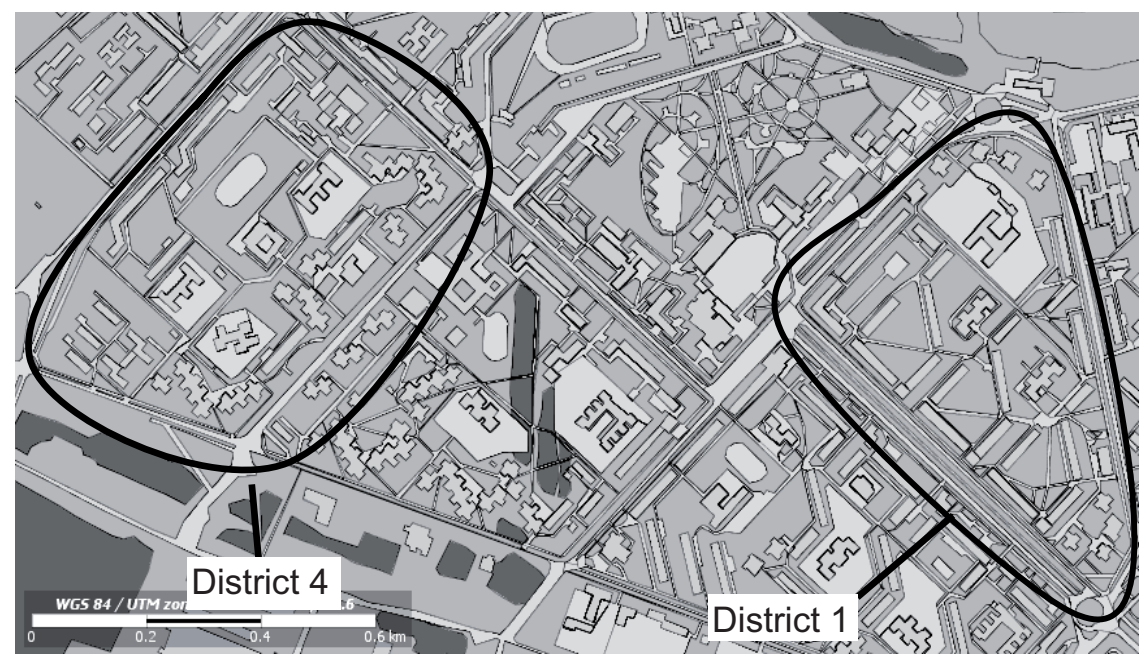

Figure 2 - The two districts identified for model comparison by the IAEA EMRAS Programme, Urban Remediation Working Group.

With this knowledge, the user must then identify the idealised environment or mix of environments that most closely resembles the real environment. Figure 2 shows the town of Pripyat that was heavily contaminated by the Chernobyl accident and that has been used in a scenario developed by the IAEA EMRAS Programme Urban Remediation Working Group for the purpose of comparing urban models such as ERMIN (Thiessen et al., 2009). In the scenario two districts of the town with high and slightly lower contamination were identified for testing. Figure 2 shows that these both contain large apartment blocks that are fairly widely spaced in grass areas with some large low buildings. The "Multi-storey block of flats opposite parkland" environment is quite appropriate to represent the apartments. The large low buildings are not so well represented in the ERMIN database although the "Industrial site" environment would have been appropriate if it was complete. In this example these areas are represented by a mix of the "Semi detached" and "open area". There is a difference between the two districts not apparent from the map in that District 1 has trees that are much more mature than District 4. ERMIN can take this into account by allowing the user to choose from a range of "parameter sets" for each idealised environment that have varying proportions of outdoor surfaces.

Figure 3 shows the dose-rate calculated; the effect of assigning grid squares to different environments can be seen with the largest doses in the high 


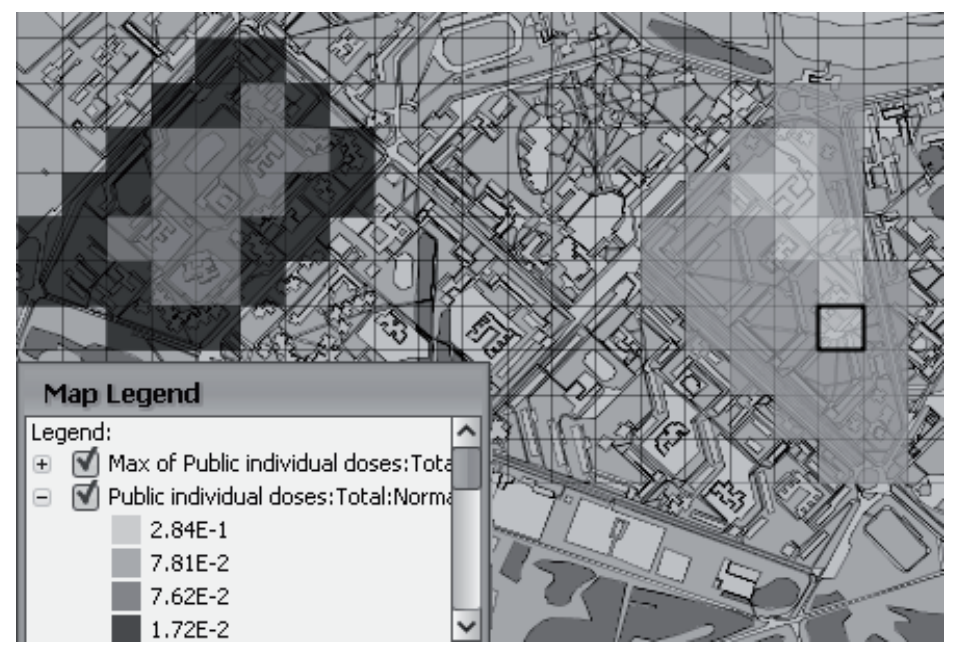

Figure 3-Results from ERMIN shown in the RODOS interface; the predicted effective doses in Sv in the first year in District 1 and District 4 of Pripyat.

contamination District 1 in the low buildings environment and the lowest doses predicted in the low contamination District 4 in the apartment zone.

An alternative approach to assigning idealised environments to regions on the basis of local knowledge is to use an agreed default environment. This will ensure consistency between operators for situations when either it is not possible to get local information or when time is too pressing - such as the early stages of an emergency.

\section{Effect of environment}

If the only effect of the built environment on the dose-rates to which the population are exposed was shielding then the developers of ERMIN could have adopted an approach that was much more simplistic than using an explicit description of the environment; for example applying location factors to a simple model of dose. However, the effect of the environment is much more subtle than simple magnitude of dose-rates. Two different environments may give initially similar dose-rates but the relative contributions from individual urban surfaces may be very different between the two environments; in one environment the roofs and walls may be the most significant contributors and in another it may be the trees. This means that a countermeasure may be effective in one environment and 

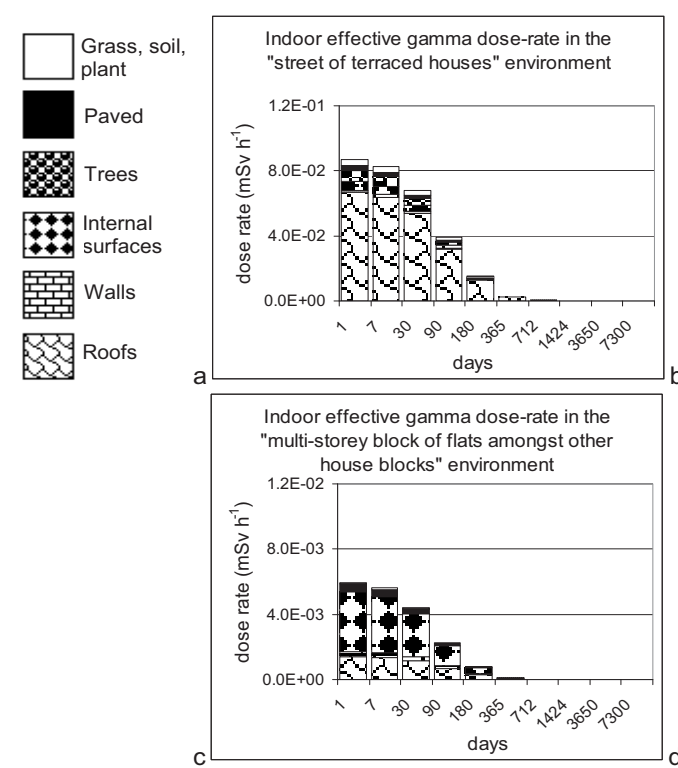
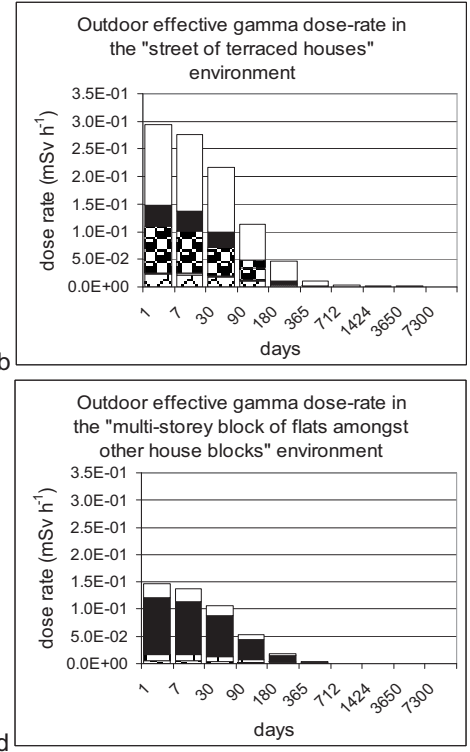

Figure 4 - (a) and (b) The predicted indoor and outdoor external effective dose-rates using the "street of terraced houses" environment and (c) and (d) the predicted indoor and outdoor dose-rates using the "multi-storey block of flats amongst other house blocks" environment.

ineffective in another. Furthermore because of the different retention characteristics of the urban surfaces, the dose-rates and the relative importance of different surfaces will vary in time. Figure 4 gives some output from ERMIN that illustrate these effects with very different surfaces contributing to the overall dose.

Another advantage of using explicit environment descriptions is that the areas of the different urban surfaces in the region can be estimated and, using values from the ERMIN database, it becomes simple to calculate the amount of work required, the cost and the amount of waste generated by any particular countermeasure. From the work required, the exposure of workers implementing the countermeasures can be estimated and from the amount of waste an estimate can be made of the activity concentration of the waste.

\section{Conclusions}

ERMIN has been released as version 1 in the RODOS and ARGOS nuclear emergency Decision Support Systems. For each run the user is required to specify 
a description of the urban environment. Although the user may find this challenging it is a fairly simple process of drawing regions on a map and assigning proportions of idealised environments from the ERMIN database to those regions. The description can be as simple as a single region encompassing the whole area of interest and assigned a single agreed default environment or it can be numerous individual specified regions with varying proportions of environments. ERMIN was designed in this way because it was recognised that the characteristics of the urban environment not only affect the dose-rates to which the population is exposed but also greatly affect the efficacy of countermeasures and so the choice of an appropriate recovery strategy.

Acknowledgements. Work on the ERMIN software received partial financial support from the European Commission Sixth Framework Programme (Nuclear Fission/Radiation Protection) under the EURANOS integrated project: European approach to nuclear and radiological emergency management and rehabilitation strategies (Contract No: FI6R-CT-2004-508843). Tatiana Duranova of VUJE, Inc., Slovak Republic supplied Figures and feedback on the use of ERMIN.

\section{REFERENCES}

Charnock T.W., Jones J.A., Singer L.N., Andersson K.G., Roed J., Thykier-Nielsen S., Mikkelsen T., Astrup P., Kaiser J.C., Müller H., Pröhl G., Raskob W., Hoe S.C., Jacobsen L.H., Shou-Jensen L., Gering F. (2009) Calculating the consequences of recovery, a European Model for Inhabited Areas. In: Proceedings International Conference of Radioecology and Environmental Radioactivity, 2008, Bergen, Norway, Radioprotection 44, 407-412.

Thiessen K.M., Arkhipov A., Batandjieva B., Charnock T.W., Gaschak S., Golikov V., Hwang W.T., Tomás J., Zlobenko B. (2009) Modelling of a large-scale urban contamination situation and remediation alternatives, J. Environ. Radioact. 100, 413-421. 